\title{
Diversity through Coded Cooperation
}

\author{
Todd E. Hunter, Student Member, IEEE and Aria Nosratinia, Member, IEEE
}

\begin{abstract}
Cooperation provides transmit diversity in cases where wireless transmitters, due to size, power, or other constraints, cannot support multiple antennas. Cooperation involves two single-antenna sources (which we call users) forming a partnership, in which each achieves space-time diversity by using their partner's antenna as a relay. We propose a new methodology, called coded cooperation, where cooperation is achieved through channel coding methods, instead of a direct relay or repetition. Each codeword is partitioned into two subsets that are transmitted from the user's and partner's antennas, respectively. Coded cooperation achieves impressive gains compared to a non-cooperative system while maintaining the same information rate, transmit power, and bandwidth. We develop tight upper bounds for bit and block error rates, which we validate through simulations. Bit and block error rate results illustrate the improvement of coded cooperation for various combinations of channel conditions between the partners and to the destination.
\end{abstract}

\section{Index Terms}

User cooperation, diversity, transmit diversity, space-time coding, wireless communications.

\section{INTRODUCTION}

Transmit diversity techniques have received much attention in recent years as a means to mitigate the detrimental effects of multi-path fading. Among the noteworthy recent discoveries in this area are trellis space-time codes [1] and block space-time codes [2], [3] for multi-element antenna arrays. Unfortunately, transmit diversity methods are not applicable to many wireless

Submitted to IEEE Transactions on Wireless Communications, February 11, 2004. This work was supported in part by the NSF under grant CCR-9985171. T. Hunter and A. Nosratinia are with the Multimedia Communications Laboratory, The University of Texas at Dallas, TX 75083-0688, USA, E-mail: \{thunter,aria\}@utdallas.edu 
systems due to size, complexity, or other constraints. A conventional example is the uplink of cellular systems, where the size of the mobile is the limiting factor. A more interesting example is ad-hoc networks and sensor networks, where size, complexity, and power are all limiting issues that preclude multiple transmit antennas.

Cooperation between pairs of wireless communication agents (which we shall call users in the remainder of this paper) has been suggested [4], [5], [6], [7] as a means to provide transmit diversity in the face of this limitation. Diversity is achieved by a signaling scheme that allows two single-antenna users to send their information using both of their antennas (Fig. 1). Sendonaris, Erkip, and Aazhang [4], [5] propose a CDMA-based two-user cooperative modulation scheme, in which each user transmits one bit over two successive symbol periods. The users transmit their own bits in the first period, then transmit their estimate of their partner's bit in the second period. Achievable rate regions and outage probabilities are examined for this cooperative modulation. Laneman and Wornell [6] compare the bit error rate (BER) for various uncoded symbol-wise detect-and-forward and amplify-and-forward cooperative protocols. Laneman, Wornell and Tse [7] extend this work by examining the outage probabilities for detectand-forward and amplify-and-forward cooperative protocols in quasi-static fading.

We present a new user cooperation methodology, called coded cooperation, in which cooperative signaling is integrated with channel coding. This method has two key characteristics. First, cooperation occurs through partitioning a user's code word such that part of the code word is transmitted by the user itself, while the remainder is transmitted by the partner through partial or complete decoding. In previous methods cooperation occurs via repetition, which may not be the best use of available bandwidth. Second, we employ error detection at the partner to avoid error propagation. Many of the previous methods either admit forwarding of erroneous estimates of the partner's symbols, or include propagation of the partner's noise. Error propagation diminishes the performance, particularly when the channel between partners is poor.

It is possible to implement these characteristics in a natural and simple manner by a method 
that uses common error control codes, as explained in the sequel. Furthermore, the incorporation of cooperation into channel coding allows a great degree of flexibility, since by varying the associated code rate, the coupling between the cooperating users can be controlled and adapted to channel conditions.

After reviewing the system model in Section II, we present the coded cooperation protocol in Section III. In Sections IV and V we develop tight upper bounds for bit and block error probability, using tools and techniques from Craig [8], Simon and Alouini [9], and Malkamäki and Leib [10]. This analysis is validated through simulations in Section VI. Performance results show that coded cooperation achieves impressive gains for a variety of channel conditions. In Section VII we discuss several practical issues, including modeling of the inter-user channel and aspects of system implementation. We conclude in Section VIII.

\section{SYSTEM MODEL}

The setup consists of two users both transmitting to a single destination. It is in the context of this multiuser communication that cooperation takes place. The channels between users (interuser channels) and from each user to the destination (uplink channels) are mutually independent and subject to flat Rayleigh fading. We consider flat fading in order to isolate the benefits of spatial diversity provided by coded cooperation, however the technique easily extends to systems that experience frequency selective fading.

The users transmit on orthogonal channels (e.g., TDMA, CDMA, or FDMA), which allows the destination, and other users in the cooperative case, to separately detect each user. In our analysis and simulations, we assume that the receivers maintain channel state information and employ coherent detection, so that we need only consider the magnitudes of the fading coefficients in the system model and analysis that follow. For BPSK modulation, the baseband-equivalent discretetime signal transmitted by User $i \in\{1,2\}$ and received by User $j \in\{0,1,2\}(j \neq i$, and $j=0$ denotes the destination) is

$$
r_{i, j}(n)=\alpha_{i, j}(n) \sqrt{E_{b, i}} \cdot b_{i}(n)+z_{j}(n)
$$


where $E_{b, i}$ is the transmitted energy per bit for user $i, b_{i}(n) \in\{-1,+1\}$ is the BPSK-modulated code bit at time $n, \alpha_{i, j}(n)$ is the fading coefficient magnitude between Users $i$ and $j$, and $z_{j}(n)$ accounts for noise and other additive interference at the receiver. We model $\alpha_{i, j}(n)$ as independent samples of a Rayleigh-distributed random variable characterized by mean-square value $\Omega_{i, j}=E_{\alpha_{i, j}}\left[\alpha_{i, j}^{2}(n)\right]$, where $E_{x}[\cdot]$ denotes the expectation operator with respect to random variable $x$. The value of $\Omega_{i, j}$ accounts for large-scale path loss and shadowing effects. For slow (quasi-static) fading, the fading coefficients remain constant $\left(\alpha_{i, j}(n)=\alpha_{i, j}\right)$ over the transmission of each source block, while for fast fading, they are i.i.d. for each transmitted symbol. The noise term $z_{j}(n)$ is modeled as independent, zero-mean additive white Gaussian noise with variance $N_{j} / 2$ (i.e., samples of a white noise process with two-sided power spectral density $N_{j} / 2$ ). We define the instantaneous received SNR for the channel between users $i$ and $j$ as

$$
\gamma_{i, j}(n)=\frac{\alpha_{i, j}^{2}(n) E_{b, i}}{N_{j}}
$$

For $\alpha_{i, j}(n)$ Rayleigh distributed, $\gamma_{i, j}(n)$ has an exponential distribution with mean

$$
\Gamma_{i, j}=E_{\alpha_{i, j}}\left[\gamma_{i, j}(n)\right]=E_{\alpha_{i, j}}\left[\frac{\alpha_{i, j}^{2}(n) E_{b, i}}{N_{j}}\right]=\Omega_{i, j} \frac{E_{b, i}}{N_{j}} .
$$

Note that for our purposes we are assuming that $\Omega_{i, j}$ and $\Gamma_{i, j}$ are constant over $n$ for a given channel, i.e., the channel statistics are not changing with time. In the analysis and performance results reported in this paper, we quantify the quality of each channel by its corresponding average received SNR as given by (3). We consider cases in which the average received SNR for the two uplink channels, $\Gamma_{1,0}$ and $\Gamma_{2,0}$, are equal (statistically similar channels) and unequal (statistically dissimilar channels). This symmetry or asymmetry results from, for example, the relative proximity of the cooperating users to each other and to the destination.

In [4], [5], [6], [7], the channels between Users $i$ and $j$ are assumed to be reciprocal, e.g., $\alpha_{i, j}(n)=\alpha_{j, i}(n)$, for the case of slow fading. The applicability of this assumption depends on the multiple access scheme and the nature of the fading experienced by the inter-user channels. In this paper we present slow fading results for two extremes: reciprocal inter-user channels, and 
those with a complete lack of reciprocity; e.g., the channels from User $i$ to User $j$ and User $j$ to User $i$ are mutually independent. We refer to these as independent inter-user channels. For the case of fast fading, we assume that the inter-user channels are always independent. We discuss further the issue of inter-user channel reciprocity in Section VII-A.

\section{CODED COOPERATION}

The users segment their source data into blocks which are augmented with a cyclic redundancy check (CRC) code, of $K$ bits per source block (including the CRC bits). Each block is then encoded with a FEC code, so that, for an overall rate $R$ code, we have $N=K / R$ total code bits per source block. Since most wireless systems already employ CRC codes, this does not represent additional overhead required by coded cooperation.

The two users cooperate by dividing the transmission of their $N$-bit code words into two successive time segments, or frames. In the first frame, each user transmits a rate $R_{1}>R$ code word with $N_{1}=K / R_{1}$ bits. This can be viewed as a subset of the total $N$ allocated code bits that contains all the original information. Each user also receives and decodes the partner's transmission. If the user successfully decodes the partner's rate $R_{1}$ code word, determined by checking the CRC bits, the user computes and transmits $N_{2}$ additional parity bits for the partner's data in the second frame, where $N_{1}+N_{2}=N$. These additional parity bits are selected such that they can be combined with the first frame code word to produce a more powerful rate $R$ code word. If the user does not successfully decode the partner, $N_{2}$ additional parity bits for the user's own data are transmitted. Each user always transmits a total of $N$ bits per source block over the two frames, and the users only transmit in their own multiple access channels. Fig. 2 illustrates the general coded cooperation framework, and Fig. 3 shows an implementation for a TDMA system. The analogous FDMA and CDMA implementations are straightforward. ${ }^{1}$

\footnotetext{
${ }^{1}$ Note that many current CDMA systems are actually hybrid CDMA/FDMA systems that use several uplink frequencies (see for example [11],[12]). Thus, we can avoid the difficulties of simultaneous transmission and reception on the same carrier, yet still preserve the advantages of CDMA, by having the partners use different carriers.
} 
We define the level of cooperation as $N_{2} / N$, the percentage of the total bits per each source block that the user transmits for his partner. A smaller percentage implies a more powerful code for the first frame and increased probability that a user successfully decodes the parter. However, this also means a smaller $N_{2}$, thus reducing gain from diversity. We examine this tradeoff as part of our evaluation of the scheme in Section VI.

In general, various channel coding methods can be used within this coded cooperation framework. For example, the overall code may be a block or convolutional code, or a combination of both. The code bits for the two frames may be partitioned through puncturing, product codes, or other forms of concatenation. To obtain the performance results given in this paper, we employ a simple but very effective implementation using rate-compatible punctured convolutional (RCPC) codes [13]. In this implementation, the overall rate $R$ code is selected from a given RCPC code family (e.g., the mother code). The code word for the first frame is obtained by applying the puncturing matrix corresponding to rate $R_{1}$, and the additional parity bits transmitted in the second frame are those punctured from the first frame.

The users act independently in the second frame, with no knowledge of whether their own first frame was correctly decoded. As a result, there are four possible cooperative cases for the transmission of the second frame, illustrated in Fig. 4. In Case 1, both users successfully decode each other, so that they each transmit for their partner in the second frame, resulting in the fully cooperative scenario depicted in Fig. 2. In Case 2, neither user successfully decodes their partner's first frame, and the system reverts to the non-cooperative case for that pair of source blocks. In Case 3, User 2 successfully decodes User 1, but User 1 does not successfully decode User 2. Consequently, neither user transmits the second set of code bits for User 2 in the second frame, but instead both transmit the second set for User 1. These two independent copies of User 1's bits are optimally combined at the destination prior to decoding. Case 4 is identical to Case 3 with the roles of User 1 and User 2 reversed. Clearly the destination must know which of these four cases has occurred in order to correctly decode the received bits. We discuss this 
issue in Section VII-B, and demonstrate that this does not significantly impact the performance or complexity of the cooperative system.

\section{PAirWise ERror Probability}

The pairwise error probability (PEP) for a coded system is defined as selecting code word $\boldsymbol{e}=[e(1), e(2), \ldots, e(N)]$ when code word $\boldsymbol{c}=[c(1), c(2), \ldots, c(N)]$ is transmitted. For a binary code with BPSK modulation, coherent detection, and maximum-likelihood decoding, the PEP conditioned on the set of instantaneous received SNR values $\gamma=[\gamma(1), \gamma(2), \ldots, \gamma(N)]$ can be written as $[9,(12.13)]$

$$
P(\boldsymbol{c} \rightarrow \boldsymbol{e} \mid \boldsymbol{\gamma})=Q\left(\sqrt{2 \sum_{n \in \eta} \gamma(n)}\right)
$$

where $Q(x)$ denotes the Gaussian $Q$-function [14, (2-1-97)]. The set $\eta$ is the set of all $n$ for which $c(n) \neq e(n)$, and the cardinality of $\eta$ is equal to the Hamming distance $d$ between code words $c$ and $e$. The selection of $e$ over $c$ is known as an error event, and thus $d$ is typically referred to as the corresponding error event Hamming weight.

For the remainder of this analysis, we restrict ourselves to the class of linear codes, where, without loss of generality, the transmitted code word $c$ can always be chosen as the all-zero code word for the purposes of error analysis. Consequently, the PEP depends only on $d$ and not the particular code words $\boldsymbol{c}$ and $\boldsymbol{e}$, so that the conditional PEP will be denoted simply by $P(d \mid \gamma)$.

\section{A. Coded Cooperation with Slow Fading}

In slow fading, the uplink channel coefficients are constant over the code word, i.e., $\alpha_{i, 0}(n)=$ $\alpha_{i, 0}$ and $\gamma_{i, 0}(n)=\gamma_{i, 0}$ constant for $n=1, \ldots, N$ for User $i$ 's uplink channel. For Case 1 (Fig. 4), when both users successfully decode each other's first frame, each user's coded bits are divided between the two user channels. Considering User 1's code word we can thus write (4) as

$$
P\left(d \mid \gamma_{1,0}, \gamma_{2,0}\right)=Q\left(\sqrt{2 d_{1} \gamma_{1,0}+2 d_{2} \gamma_{2,0}}\right)
$$


where $d_{1}$ and $d_{2}$ are the portions of the error event bits transmitted through User 1's and User 2's channel respectively, such that $d_{1}+d_{2}=d$. Note that $d_{1}$ and $d_{2}$ are independent of $\gamma_{1,0}$ and $\gamma_{2,0}$.

To obtain the unconditional PEP we must average (5) over the fading distributions, as

$$
P(d)=\int_{0}^{\infty} \int_{0}^{\infty} P\left(d \mid \gamma_{1,0}, \gamma_{2,0}\right) p\left(\gamma_{1,0}\right) p\left(\gamma_{2,0}\right) d \gamma_{1,0} d \gamma_{2,0}
$$

where $p(x)$ is the probability density function of random variable $x$. We can obtain an exact solution to (6) using the techniques of Simon and Alouini [9]. The first step is to use the following alternative representation for the Gaussian $Q$-function, originally derived by Craig [8], and then applied to performance analysis in fading channels in [9]

$$
Q(x)=\frac{1}{\pi} \int_{0}^{\pi / 2} \exp \left(-\frac{x^{2}}{2 \sin ^{2} \theta}\right) d \theta, \quad x \geq 0 .
$$

Using (7) in (5) and (6) gives

$$
P(d)=\frac{1}{\pi} \int_{0}^{\pi / 2}\left[\int_{0}^{\infty} \exp \left(-\frac{d_{1} \gamma_{1,0}}{\sin ^{2} \theta}\right) p\left(\gamma_{1,0}\right) d \gamma_{1,0}\right]\left[\int_{0}^{\infty} \exp \left(-\frac{d_{2} \gamma_{2,0}}{\sin ^{2} \theta}\right) p\left(\gamma_{2,0}\right) d \gamma_{2,0}\right] d \theta
$$

The two inner integrals in (8) have the form of moment-generating functions for the two densities $p\left(\gamma_{1,0}\right)$ and $p\left(\gamma_{2,0}\right)[15,(3.5-1)-(3.5-3)]$. We can thus evaluate these integrals using the techniques of [9] to obtain, for Rayleigh fading,

$$
P(d)=\frac{1}{\pi} \int_{0}^{\pi / 2}\left(1+\frac{d_{1} \Gamma_{1,0}}{\sin ^{2} \theta}\right)^{-1}\left(1+\frac{d_{2} \Gamma_{2,0}}{\sin ^{2} \theta}\right)^{-1} d \theta .
$$

We can obtain a closed-form expression for this expression using [9, (5A.58)-(5A.60)], but it provides no additional insight for coded cooperation, so we omit it here. This probability is upper bounded by:

$$
P(d) \leq \frac{1}{2}\left(\frac{1}{1+d_{1} \Gamma_{1,0}}\right)\left(\frac{1}{1+d_{2} \Gamma_{2,0}}\right) .
$$

For large SNR, the PEP is inversely proportional to the product of the average SNR of the uplink channels. Thus, if $d_{1}$ and $d_{2}$ are both non-zero, full diversity order of two is achieved when both partners successfully receive each other and cooperate. This is a significant improvement over no cooperation, which is fundamentally limited to diversity order of one (see (20)). 
For Case 3, where User 1 does not successfully decode User 2, but User 2 successfully decodes User 1, both users send the same additional parity bits for User 1 in the second frame. These bits are optimally combined at the destination, so that the conditional PEP (5) for User 1 becomes

$$
P\left(d \mid \gamma_{1,0}, \gamma_{2,0}\right)=Q\left(\sqrt{2 d_{1} \gamma_{1,0}+2 d_{2}\left(\gamma_{1,0}+\gamma_{2,0}\right)}\right)=Q\left(\sqrt{2 d \gamma_{1,0}+2 d_{2} \gamma_{2,0}}\right)
$$

and the unconditional PEP becomes

$$
P(d)=\frac{1}{\pi} \int_{0}^{\pi / 2}\left(1+\frac{d \Gamma_{1,0}}{\sin ^{2} \theta}\right)^{-1}\left(1+\frac{d_{2} \Gamma_{2,0}}{\sin ^{2} \theta}\right)^{-1} d \theta \leq \frac{1}{2}\left(\frac{1}{1+d \Gamma_{1,0}}\right)\left(\frac{1}{1+d_{2} \Gamma_{2,0}}\right) .
$$

Equation (12) illustrates that User 1 again achieves full diversity order two for Case 3.

\section{B. Coded Cooperation with Fast Fading}

For fast fading, the fading coefficients are no longer constant over the code word, but are i.i.d. across the coded bits. Thus, for Case 1, we generalize (4) as

$$
P\left(d \mid \gamma_{1,0}, \gamma_{2,0}\right)=Q\left(\sqrt{2 \sum_{n \in \eta_{1}} \gamma_{1,0}(n)+2 \sum_{n \in \eta_{2}} \gamma_{2,0}(n)}\right)
$$

where the set $\eta_{i}$ is the portion of the $d$ error event bits transmitted through User $i$ 's channel. The cardinalities of $\eta_{1}$ and $\eta_{2}$ are $d_{1}$ and $d_{2}$ respectively, where again $d_{1}+d_{2}=d$, and $d_{1}$ and $d_{2}$ are independent of $\gamma_{1,0}(n)$ and $\gamma_{2,0}(n)$ for all $n$.

Averaging over the fading to obtain the unconditional PEP now involves a $d$-fold integration, for which the techniques of [9] again provide a tractable solution. Applying (7) gives the following integral expression for unconditional PEP

$$
\begin{aligned}
P(d) & =\frac{1}{\pi} \int_{0}^{\pi / 2} \prod_{n \in \eta_{1}}\left[\int_{0}^{\infty} \exp \left(-\frac{\gamma_{1,0}(n)}{\sin ^{2} \theta}\right) p\left(\gamma_{1,0}(n)\right) d \gamma_{1,0}(n)\right] \\
& \times \prod_{n \in \eta_{2}}\left[\int_{0}^{\infty} \exp \left(-\frac{\gamma_{2,0}(n)}{\sin ^{2} \theta}\right) p\left(\gamma_{2,0}(n)\right) d \gamma_{2,0}(n)\right] d \theta
\end{aligned}
$$

Each inner integral in (14) has the same form as in (8), so that for Rayleigh fading we obtain

$$
P(d)=\frac{1}{\pi} \int_{0}^{\pi / 2}\left[\prod_{n \in \eta_{1}}\left(1+\frac{\Gamma_{1,0}}{\sin ^{2} \theta}\right)^{-1}\right]\left[\prod_{n \in \eta_{2}}\left(1+\frac{\Gamma_{2,0}}{\sin ^{2} \theta}\right)^{-1}\right] d \theta .
$$


Applying our assumption that $\Gamma_{1,0}$ and $\Gamma_{2,0}$ are constant over $n$ results in

$$
P(d)=\frac{1}{\pi} \int_{0}^{\pi / 2}\left(1+\frac{\Gamma_{1,0}}{\sin ^{2} \theta}\right)^{-d_{1}}\left(1+\frac{\Gamma_{2,0}}{\sin ^{2} \theta}\right)^{-d_{2}} d \theta \leq \frac{1}{2}\left(\frac{1}{1+\Gamma_{1,0}}\right)^{d_{1}}\left(\frac{1}{1+\Gamma_{2,0}}\right)^{d_{2}} .
$$

Again we have in (16) an exact expression for the unconditional PEP that is easily evaluated via numerical techniques (we can obtain a closed-form expression for the integral in (16) from [9, (5A.58)-(5A.60)], but again it gives no further insight to coded cooperation).

Equation (16) shows that the diversity order for fast fading is equal to the total Hamming weight $d=d_{1}+d_{2}$. This is also true for no cooperation (see (21)). For statistically dissimilar uplink channels $\left(\Gamma_{1,0} \neq \Gamma_{2,0}\right)$, (16) indicates definite improvement for the user with the lower uplink average SNR, which is an important practical result. For statistically similar uplink channels, (16) becomes equal to (21). Intuitively we see that coded cooperation does not provide additional diversity when the average uplink SNR are equal.

For Case 3 the conditional PEP (13) for User 1 becomes

$$
\begin{aligned}
P\left(d \mid \gamma_{1,0}, \gamma_{1,0}\right) & =Q\left(\sqrt{2 \sum_{n \in \eta_{1}} \gamma_{1,0}(n)+2 \sum_{n \in \eta_{2}} \gamma_{1,0}(n)+2 \sum_{n \in \eta_{2}} \gamma_{2,0}(n)}\right) \\
& =Q\left(\sqrt{2 \sum_{n \in \eta} \gamma_{1,0}(n)+2 \sum_{n \in \eta_{2}} \gamma_{2,0}(n)}\right)
\end{aligned}
$$

and unconditional PEP becomes

$$
P(d)=\frac{1}{\pi} \int_{0}^{\pi / 2}\left(1+\frac{\Gamma_{1,0}}{\sin ^{2} \theta}\right)^{-d}\left(1+\frac{\Gamma_{2,0}}{\sin ^{2} \theta}\right)^{-d_{2}} d \theta \leq \frac{1}{2}\left(\frac{1}{1+\Gamma_{1,0}}\right)^{d}\left(\frac{1}{1+\Gamma_{2,0}}\right)^{d_{2}} .
$$

Equation (18) shows that User 1 does achieve improved diversity $\left(d+d_{2}\right.$ vs. $\left.d\right)$ for Case 3 .

\section{No Cooperation}

For non-cooperative transmission in slow fading, all the code bits for a user are transmitted through the same channel (e.g., $d_{1}=d$ and $d_{2}=0$ ). Thus for slow fading we have conditional and unconditional PEP

$$
P(d \mid \gamma)=Q(\sqrt{2 d \gamma})
$$




$$
P(d)=\frac{1}{\pi} \int_{0}^{\pi / 2}\left(1+\frac{d \Gamma}{\sin ^{2} \theta}\right)^{-1} d \theta \leq \frac{1}{2}\left(\frac{1}{1+d \Gamma}\right) .
$$

For fast fading, the conditional PEP is given by (4), and the unconditional PEP is

$$
P(d)=\frac{1}{\pi} \int_{0}^{\pi / 2}\left(1+\frac{\Gamma}{\sin ^{2} \theta}\right)^{-d} d \theta \leq \frac{1}{2}\left(\frac{1}{1+\Gamma}\right)^{d} .
$$

These are similar to the results reported in, for example, [13, (13)], [14, (14-3-7)]. They are included here for reference because they are used in Section V-B to compute the end-to-end performance of coded cooperation. Specifically, for Case 2, the PEP for both users corresponds to non-cooperative transmission with the overall rate $R$ code word. For Cases 3 and 4 , the PEP for the user that is not decoded correctly by the partner corresponds to non-cooperative transmission with the rate $R_{1}$ code word used for transmission of the first frame.

\section{Bit AND Block ERror Rate ANALYSis}

We now use the above PEP results to determine the end-to-end bit and block error probabilities for coded cooperation. The first step is calculating the probabilities of the cooperative cases.

\section{A. Cooperative Case Probabilities}

The cooperative case probabilities are determined by the BLER of the first frame transmission. The BLER for a terminated convolutional code is bounded by [10, (12)], [16, (11)]

$$
P_{\text {block }}(\boldsymbol{\gamma}) \leq 1-\left(1-P_{E}(\boldsymbol{\gamma})\right)^{B} \leq B \cdot P_{E}(\boldsymbol{\gamma})
$$

where $B$ is the number of trellis branches in the code word, and $P_{E}(\gamma)$ is the error event probability conditioned on $\gamma$, the vector state of the channel. $P_{E}$ is bounded as [17, (4.4.5)]

$$
P_{E}(\gamma) \leq \sum_{d=d_{f}}^{\infty} a(d) P(d \mid \gamma)
$$

where $d_{f}$ is the code free distance and $a(d)$ is the number of error events of Hamming weight $d$. 
We parameterize the four cases by $\Theta \in\{1,2,3,4\}$ and we can express the conditional probability for Case $1(\Theta=1)$ as follows (bounds for the other cases are developed similarly):

$$
\begin{aligned}
P\left(\Theta=1 \mid \gamma_{1,2}, \boldsymbol{\gamma}_{2,1}\right) & =\left(1-P_{b l o c k, 1}\left(\boldsymbol{\gamma}_{1,2}\right)\right)\left(1-P_{b l o c k, 2}\left(\boldsymbol{\gamma}_{2,1}\right)\right) \\
& \geq\left(1-P_{E, 1}\left(\boldsymbol{\gamma}_{1,2}\right)\right)^{B}\left(1-P_{E, 2}\left(\boldsymbol{\gamma}_{2,1}\right)\right)^{B} \\
& \geq\left(1-B P_{E, 1}\left(\boldsymbol{\gamma}_{1,2}\right)\right)\left(1-B P_{E, 1}\left(\boldsymbol{\gamma}_{2,1}\right)\right)
\end{aligned}
$$

To calculate end-to-end error probabilities, we need the unconditional probability $P(\Theta)$ :

$$
P(\Theta)=\int_{\boldsymbol{\gamma}_{1,2}} \int_{\boldsymbol{\gamma}_{2,1}} P\left(\Theta \mid \boldsymbol{\gamma}_{1,2}, \boldsymbol{\gamma}_{2,1}\right) p\left(\boldsymbol{\gamma}_{1,2}\right) p\left(\boldsymbol{\gamma}_{2,1}\right) d \boldsymbol{\gamma}_{1,2} d \boldsymbol{\gamma}_{2,1}
$$

For slow fading, vectors $\gamma_{1,2}$ and $\gamma_{2,1}$ reduce to scalars $\gamma_{1,2}$ and $\gamma_{2,1}$. For reciprocal inter-user channels, $\gamma_{1,2}=\gamma_{2,1}$, and $P\left(\Theta \mid \gamma_{1,2}\right)$ is conditioned on a single variable, reducing (25) to a single integral. For independent inter-user channels, the unconditional first-frame BLER of the two users are independent, and $P(\Theta)$ has form analogous to the first line of (24), e.g., for Case 1,

$$
P(\Theta=1)=\left(1-P_{b l o c k, 1}\right) \cdot\left(1-P_{b l o c k, 2}\right) .
$$

To obtain tight bounds for the case of slow fading, we use the limit-before-average technique from [10] with the appropriate conditional (on fading) PEP to evaluate (25) and (26). For example, for Case 1 with reciprocal inter-user channels we have

$$
\begin{aligned}
P(\Theta=1) & \geq \int_{0}^{\infty}\left(1-\min \left[1, \sum_{d=d_{f}}^{\infty} a(d) P\left(d \mid \gamma_{1,2}\right)\right]\right)^{B} \\
& \times\left(1-\min \left[1, \sum_{d=d_{f}}^{\infty} a(d) P\left(d \mid \gamma_{1,2}\right)\right]\right)^{B} p\left(\gamma_{1,2}\right) d \gamma_{1,2}
\end{aligned}
$$

With independent inter-user channels, we compute the unconditional BLER for User $i$ as

$$
P_{\text {block }, i} \leq 1-\int_{0}^{\infty}\left(1-\min \left[1, \sum_{d=d_{f}}^{\infty} a(d) P\left(d \mid \gamma_{i, j}\right)\right]\right)^{B} p\left(\gamma_{i, j}\right) d \gamma_{i, j}
$$


and apply the results to (26). The unconditional probabilities for the other cases are evaluated similarly. Note that (27) and (28) must be computed numerically due to the minimization.

Sufficiently tight bounds for fast fading are obtained using the unconditional PEP directly in (23) and (22) [10]. Since we assume independent inter-user channels for fast fading, we can apply these results to (26) for Case 1, and the corresponding expressions for the other cases.

\section{B. End-to-End Error Analysis}

The overall end-to-end unconditional BER is equal to the average of the unconditional BER over the four possible transmission scenarios discussed in Section V-A as

$$
P_{b}=\sum_{i=1}^{4} P_{b}(\Theta) P(\Theta=i)
$$

The end-to-end BLER has a similar expression.

The conditional BLER is given by (22)-(23), and the conditional BER is bounded by [17,

$$
P_{b}(\boldsymbol{\gamma}, \Theta) \leq \frac{1}{k_{c}} \sum_{d=d_{f}}^{\infty} c(d) P(d \mid \boldsymbol{\gamma}, \Theta)
$$

where $c(d)$ is the number of information bit errors for code words or error events with Hamming weight $d$, and $k_{c}$ is the number of input bits for each branch of the code trellis.

We again use the limit-before-average technique [10] with the appropriate conditional PEP expressions to obtain tight bounds for slow fading. The unconditional BER and BLER are

$$
\begin{gathered}
P_{b}(\Theta) \leq \int_{0}^{\infty} \int_{0}^{\infty} \min \left[\frac{1}{2}, \frac{1}{k_{c}} \sum_{d=d_{f}}^{\infty} c(d) P\left(d \mid \gamma_{1,0}, \gamma_{2,0}, \Theta\right)\right] p\left(\gamma_{1,0}\right) p\left(\gamma_{2,0}\right) d \gamma_{1,0} d \gamma_{2,0} \\
P_{\text {block }}(\Theta) \leq 1-\int_{0}^{\infty} \int_{0}^{\infty}\left(1-\min \left[1, \sum_{d=d_{f}}^{\infty} a(d) P\left(d \mid \gamma_{1,0}, \gamma_{2,0}, \Theta\right)\right]\right)^{B} \\
\cdot p\left(\gamma_{1,0}\right) p\left(\gamma_{2,0}\right) d \gamma_{1,0} d \gamma_{2,0} .
\end{gathered}
$$


For fast fading, tight bounds are obtained using the unconditional (on fading) $P(d \mid \Theta)$ expression directly in the summation (23) or (30), in lieu of computing (31) [10]. Applying these and the previous results to (29) gives upper bounds for the end-to-end bit and block error probabilities. (Although the case probabilities (Section V-A) are not all strictly upper bounds, i.e. (24) and (27), the net effect is that the cases with lower error rate are weighted less than their true probabilities, and those with higher error rate are weighted more, resulting in an overall upper bound for (29).)

Whenever both users cooperate (Case 1) each user's message sees two independent fading paths and a diversity order of two is achieved. When a user's message does not benefit from cooperation the diversity is one. Therefore, the overall diversity order, interpreted as the slope of the error rate curve, is the average of the diversities in the four cases, weighted by the probabilities of the four cases. These probabilities are determined by the inter-user channel conditions. At high inter-user SNR, Case 1 is dominant and coded cooperation achieves full diversity order of two. ${ }^{2}$ We note that in order to operate at realistic SNR's, some of our simulations are not in this dominant mode, and for that reason some of the simulations show diversity less than two.

\section{Vi. Performance Evaluation}

To evaluate the performance, we implement coded cooperation using RCPC codes as described in Section III. Specifically, we use the family of RCPC codes with memory $M=4$, puncturing period $P=8$, and rate $1 / 4$ mother code given by Hagenauer [13]. For slow fading, we choose overall code rate $R=1 / 4$, while for fast fading we use $R=2 / 5$. In all cases, the source block size is $K=128$ bits. We computed via computer enumeration the distance spectra $a(d)$ and $c(d)$, including the partitioning of the Hamming weight $d$ into $d_{1}$ and $d_{2}$. For the simulations, we

\footnotetext{
${ }^{2}$ For any fixed set of probabilities, the errors of diversity order one will eventually dominate at high enough uplink SNR (even though such SNR's may be unrealistic in practice). Strictly speaking, to achieve diversity order of two, the ratio of the case probabilities in the asymptote must keep up with the increased uplink SNR. Therefore to make the above statement more precise, one more condition must be added. For example, one might say: "diversity of two is achieved if a fixed uplink to inter-user SNR ratio is maintained in the asymptote."
} 
use a 16-bit CRC code with generator polynomial given by coefficients 15935 (hexadecimal notation). For our analysis we assume perfect error detection. Figures 5 through 9 present analytical and simulation results for various scenarios described in more detail below. Due to space considerations, we only show results for BER; results for BLER are similar. Since all comparisons are between systems with equal information rate $K$ bits per source block, and equal code rate $R$, we plot the BER versus the channel SNR. Plotting BER versus the information bit SNR yields identical results, with the $x$-axis values shifted by $10 \log R \mathrm{~dB}$.

\section{A. Slow Fading}

Fig. 5 shows the BER for slow fading with reciprocal inter-user channels of various qualities. The users have statistically similar uplink channels $\left(\Gamma_{1,0}=\Gamma_{2,0}\right)$, and the level of cooperation is $50 \%$. We see that coded cooperation with a perfect inter-user channel performs virtually identically to a comparable two-antenna transmit diversity system. In this system, we use as an outer code the same rate $1 / 4$ RCPC code that we use for coded cooperation, concatenated with the space-time block code proposed by Alamouti [2]. Since the Alamouti code is known to provide full diversity order two [2], this result confirms the PEP analysis from Section IV-A and demonstrates that coded cooperation does achieve full diversity for Case 1. The improvement over no cooperation remains dramatic for good inter-user channels. For example, when the inter-

user channel has $10 \mathrm{~dB}$ average SNR, the gain is about $9 \mathrm{~dB}$ for BER $10^{-3}$. The gain decreases as the inter-user channel worsens, as well as the curve slope as discussed at the end of the previous section. However, coded cooperation still achieves significant gain even when the interuser channel is much worse that the uplink channels. For example, we see a 2-3dB gain for an inter-user channel with average SNR 0dB over the range of 0-20dB average uplink SNR.

Fig. 6 compares the performance of coded cooperation at $50 \%$ and $25 \%$, for both a perfect inter-user channel and one with average SNR of 10dB. The user uplink channels again have equal average SNR. When the inter-user channel is perfect, both users always cooperate (we have Case 1 exclusively), and 50\% cooperation yields better performance. This is predicted by 
the PEP of (10), since we expect the product $d_{1} \cdot d_{2}$ to be maximized for $50 \%$ cooperation $\left(d_{1}\right.$ and $d_{2}$ should be approximately equal). However, as the inter-user channel becomes worse, the situation changes. Fig. 6 shows that $25 \%$ cooperation becomes better than $50 \%$ cooperation for the $10 \mathrm{~dB}$ inter-user channel, by as much as $2 \mathrm{~dB}$ for higher uplink SNR. For poor inter-user channels, a stronger code in the first frame is more important to the overall performance than maximizing the product $d_{1} \cdot d_{2}$. This is a result of averaging over the four cooperative cases.

In Fig. 7, we examine the performance of coded cooperation when the users have statistically dissimilar uplink channels. We fix the average uplink SNR for User 1 at 20dB, while varying User 2's average uplink SNR from $0 \mathrm{~dB}$ to $20 \mathrm{~dB}$. The inter-user channel has average SNR 10dB. Fig. 7 shows that User 2, with the worse uplink channel, improves dramatically with coded cooperation, exhibiting a gain of $11-13 \mathrm{~dB}$ relative to no cooperation. More interestingly however, User 1, with the better uplink channel, also achieves a marked improvement in performance by cooperating, a result that is not necessarily intuitive. Thus, even a user with a very good uplink channel has a strong motivation to cooperation in a slow-fading environment.

In order to simplify the plots, we show in Figures 5 and 7 the analytical bounds truncated using only the first few terms of the distance spectrum, which is sufficient for our purposes. Because of this approximation, these bounds appear slightly tighter than that of [10]. Using all of the terms gives tight upper bounds with convergence behavior similar to [10].

\section{B. Comparison with Amplify-and-Forward}

In this section we compare coded cooperation with a coded version of the amplify-and-forward protocol proposed in [6], [7]. In this scheme, the coded bits of each user are amplified and forwarded in the analog domain by the partner. This comparison serves to illustrate the advantages of coded cooperation over previously proposed repetition-based schemes.

We implement the amplify-and-forward protocol with a rate- $1 / 2$ convolutional code, resulting in an overall rate of $1 / 4$. The overall code rate for coded cooperation is also $R=1 / 4$. Fig. 8 shows the comparison of simulated BER for slow Rayleigh fading, equal uplink average SNR, 
and inter-user channels with $10 \mathrm{~dB}$ and $0 \mathrm{~dB}$ average SNR. Coded cooperation maintains an edge of up to 1-2dB over amplify-and-forward, depending on the uplink SNR. The level of cooperation that achieves the best performance for coded cooperation varies between $50 \%$ and $25 \%$, depending on the channel conditions. The level of cooperation for amplify-and-forward, of course, is inflexible (set at $50 \%$ ) since repetition is a core part of that protocol.

\section{Fast Fading}

Based on the results of Section IV-B, we focus our attention for fast fading on the case when the two users have statistically dissimilar uplink channels. In Fig. 9, the average uplink SNR for User 1 is fixed at $10 \mathrm{~dB}$, while User 2's varies from $0 \mathrm{~dB}$ to $10 \mathrm{~dB}$. The inter-user channels have average SNR of $10 \mathrm{~dB}$, and are assumed to be independent. Recall that we use overall code rate $R=2 / 5$ for fast-fading. Under these conditions, 30\% cooperation provides the best performance. Fig. 9 shows that the bounds developed in Sections IV and V are quite tight. User 2, with the worse uplink channel, experiences significant improvement, gaining up to 33.5dB at low uplink SNR. User 1, with the better uplink channel, sacrifices some performance by cooperating. Nevertheless, even in fast fading, coded cooperation can help a user with a poor uplink channel, provided that the partner's performance remains acceptable. This is an important practical result with respect to maintaining a minimum system-wide quality of service.

\section{IMPLEMENTATION ISSUES}

\section{A. Inter-user Channel Reciprocity and Multiple Access Protocol}

As discussed in Section II, previous works on user cooperation ([4], [5], [6], [7]) generally assume that the channels between the users are reciprocal for slow fading. This is justifiable for TDMA systems and CDMA systems, but less so for FDMA systems. In FDMA, adjacent frequency channels generally may not have identical fading. However, it is likely that they are correlated to some degree, and are not fully independent. The following results show that even 
if we assume the extreme condition of fully independent inter-user channels, the gains of coded cooperation can be maintained via judicious choice of cooperation level.

Fig. 10 shows the difference in analytical BER bounds for reciprocal versus independent interuser channels of various qualities. All channels are subject to slow Rayleigh fading, and the user uplink channels are statistically similar. For 50\% cooperation, having independent inter-user channels reduces the gains by $1-3 \mathrm{~dB}$, However, for $25 \%$ cooperation, the results for reciprocal and independent inter-user channels are well within $1 \mathrm{~dB}$ of each other.

For reciprocal channels, it is more likely that both users will act similarly, i.e., both will either decode each other successfully or unsuccessfully. This symmetric behavior leads to (slightly) better performance with reciprocal channels. The stronger first-frame code employed with $25 \%$ cooperation reduces the effect of the more asymmetric behavior with independent inter-user channels. This points to one of the benefits of the flexibility available in the framework of coded cooperation: the ability to vary the code rates between the first and second frames provides a degree of robustness to unfavorable conditions.

For fast fading, the inter-user channels will clearly be independent unless the first frame transmissions of the users are exactly synchronized. Therefore all the fast fading experiments in this paper assume independent inter-user channels.

\section{B. Cooperative Overhead}

Section III notes that the destination must know which of the four cooperative cases has occurred in order to correctly decode the received frames. One approach is that each user sends one additional bit in the second frame to indicate the first-frame decision. This bit would need error protection, leading to a tradeoff between the rate and the probability of error for this bit.

An alternative approach is to have the destination simply decode according to each of the four cases in succession, according to their relative probabilities of occurrence, until the CRC code indicates correct decoding. This strategy maintains the overall system performance and rate at the cost of some added complexity at the destination. We can express this complexity in terms of 
the expected number of decoding attempts $k$ required by the destination to process the received frames for a given code word. The complexity factor $k$ can be expressed as

$$
k=4 \cdot P_{\text {block }}+\left(1-P_{\text {block }}\right) \sum_{\Theta=1}^{4} \Theta \cdot P(\Theta),
$$

where $P_{\text {block }}$ denotes the end-to-end BLER for the code word, and $P(\Theta)$ denotes the probability of Case $\Theta$, with index $\Theta$ ordered such that $\Theta=1$ corresponds to the most probable case. For end-to-end BLER $10^{-2}$, Table I gives $k$ values for $50 \%$ cooperation in a reciprocal inter-user channel, which show that the increase in complexity at the destination is minimal.

\section{CONCLUSIONS AND RELATED WORK}

We present a new technique for wireless user cooperation, in which cooperation is integrated with channel coding. Diversity is achieved by partitioning a user's code word into two parts. Each user receives the first codeword partition from the partner, and upon successful decoding (determined via a CRC code), transmits the second codeword partition. The two partitions are thus received at the destination through independent fading channels. This coded cooperation framework may be implemented using block or convolutional codes, and various methods of partitioning the code words (puncturing, product codes, parallel and serial concatenation, etc). Our examples in this paper use RCPC codes and partition the code words via puncturing.

We develop tight upper bounds for the performance of coded cooperation, and validate these bounds with simulations. For slow Rayleigh fading, coded cooperation yields impressive gains for both partners, even when the inter-user channel is much worse than the uplink channels, and when one has a much worse uplink channel than the other. For fast i.i.d. Rayleigh fading, coded cooperation provides important gains when the users have different uplink channel qualities. The user with the worse uplink channel experiences significant improvement under these conditions.

In a subsequent work [18], we consider two extensions to the coded cooperation framework developed in this paper. The first uses space-time signaling concepts to improve the performance in fast fading. The second extension involves implementing coded cooperation using turbo codes. 


\section{REFERENCES}

[1] V. Tarokh, N. Seshadri, and A. R. Calderbank, "Space-time codes for high data rate wireless communication: Performance criteria and code construction," IEEE Trans. Inform. Theory, vol. 44, no. 2, pp. 744-765, March 1998.

[2] S. M. Alamouti, "A simple transmit diversity technique for wireless communications," IEEE J. Select. Areas Commun., vol. 16, no. 8, pp. 1451-1458, October 1998.

[3] V. Tarokh, H. Jafarkhani, and A. R. Calderbank, "Space-time block codes from orthogonal designs," IEEE Trans. Inform. Theory, vol. 45, no. 5, pp. 1456-1467, July 1999.

[4] A. Sendonaris, E. Erkip, and B. Aazhang, “User cooperation diversity-Part I: System description,” IEEE Trans. Commun., vol. 51, no. 11, pp. 1927-1938, November 2003.

[5] — , "User cooperation diversity-Part II: Implementation aspects and performance analysis," IEEE Trans. Commun., vol. 51, no. 11, pp. 1939-1948, November 2003.

[6] J. N. Laneman and G. W. Wornell, "Energy-efficient antenna sharing and relaying for wireless networks," in Proc. IEEE Wireless Communcations and Networking Conference (WCNC), Chicago, IL, September 2000, pp. 7-12.

[7] J. N. Laneman, G. W. Wornell, and D. N. C. Tse, "An efficient protocol for realizing cooperative diversity in wireless networks," in Proc. IEEE International Symposium on Information Theory (ISIT), Washington, D. C., June 2001, p. 294.

[8] J. W. Craig, "A new, simple, and exact result for calculating the probability of error for two-dimensional signal constellations," in Proc. IEEE MILCOM, McLean, VA, October 1991, pp. 571-575.

[9] M. K. Simon and M.-S. Alouini, Digital Communication over Fading Channels: A Unified Approach to Performance Analysis. New York: John Wiley and Sons, 2000.

[10] E. Malkamäki and H. Leib, "Evaluating the performance of convolutional codes over block fading channels," IEEE Trans. Inform. Theory, vol. 45, no. 5, pp. 1643-1646, July 1999.

[11] E. I. S.-. (IS-95), "Mobile station - base station compatibility standard for dual-mode wideband spread spectrum celluar system."

[12] 3rd Generation Partnership Project 3G TS 25.101, "UE radio transmission and reception (FDD)."

[13] J. Hagenauer, "Rate-compatible punctured convolutional codes (RCPC codes) and their applications," IEEE Trans. Commun., vol. 36, no. 4, pp. 389-400, April 1988.

[14] J. G. Proakis, Digital Communications, 3rd ed. New York: McGraw-Hill, 1995.

[15] H. Stark and J. W. Woods, Probability, Random Processes, and Estimation Theory for Engineers, 1st ed. Englewood Cliffs, NJ: Prentice Hall, 1986.

[16] S. Kallel and C. Leung, "Efficient ARQ schemes with multiple copy decoding," IEEE Trans. Commun., vol. 40, pp. 642-650, March 1992.

[17] A. Viterbi and J. K. Omura, Principles of Digital Communcation and Coding. New York: McGraw-Hill, 1979.

[18] M. Janani, A. Hedayat, T. E. Hunter, and A. Nosratinia, "Coded cooperation in wireless communications: Space-time transmission and iterative decoding," IEEE Trans. Signal Processing, vol. 52, no. 2, pp. 362 - 371, Feb. 2004. 


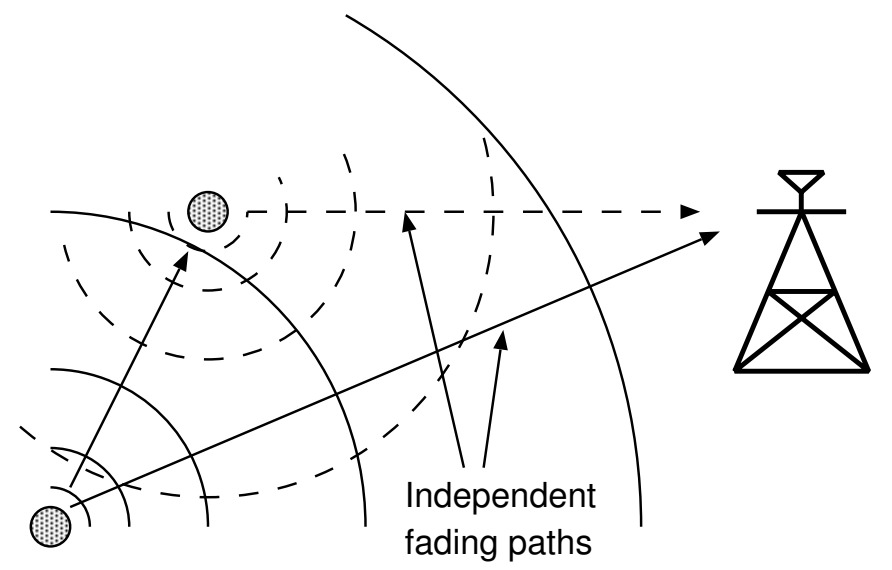

Fig. 1. Cooperation diversity

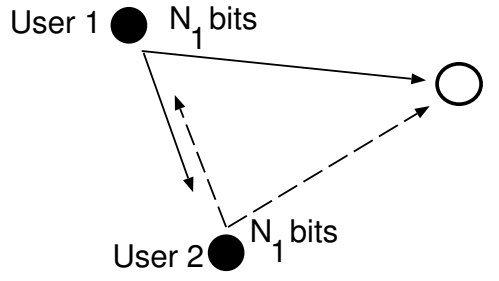

Frame 1

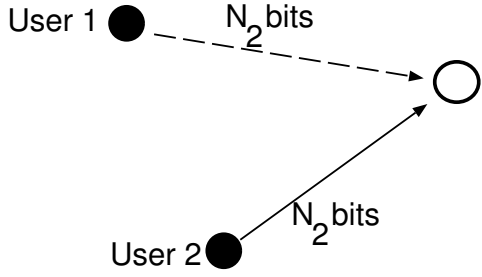

Frame 2

Fig. 2. Cooperative transmission scheme.

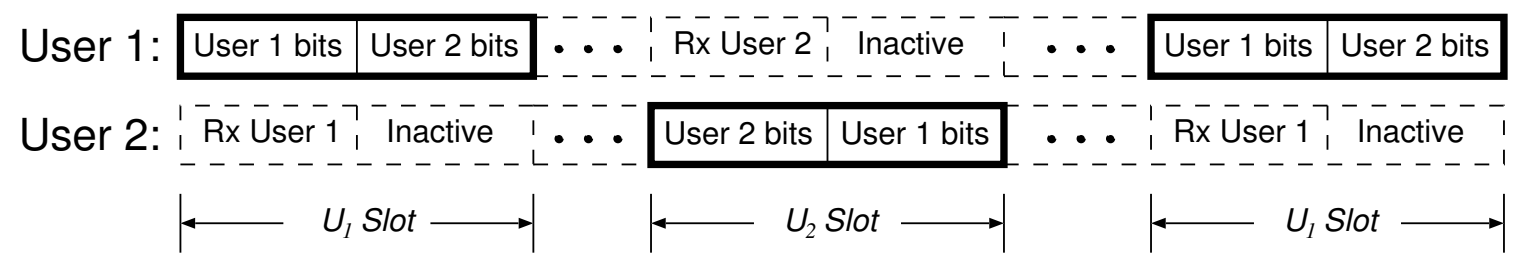

Fig. 3. Coded cooperation implementation for a system using TDMA. 


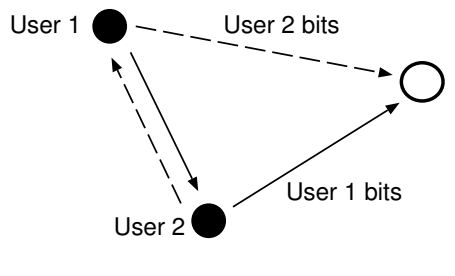

Case 1

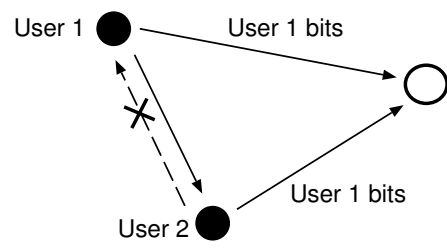

Case 3
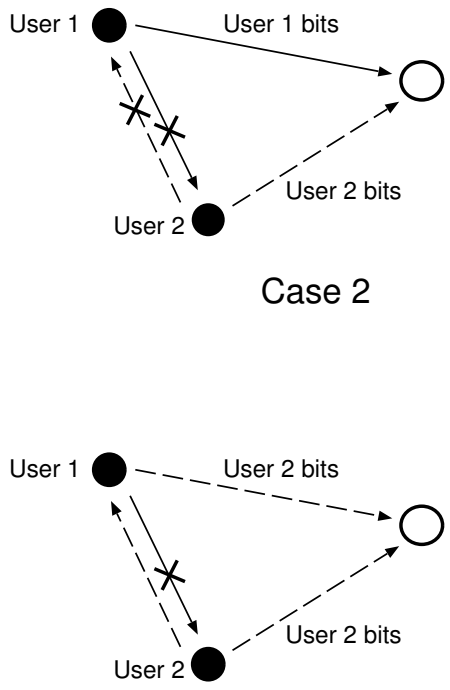

Case 4

Fig. 4. Four cooperative cases for second frame transmission based on the first frame decoding results.

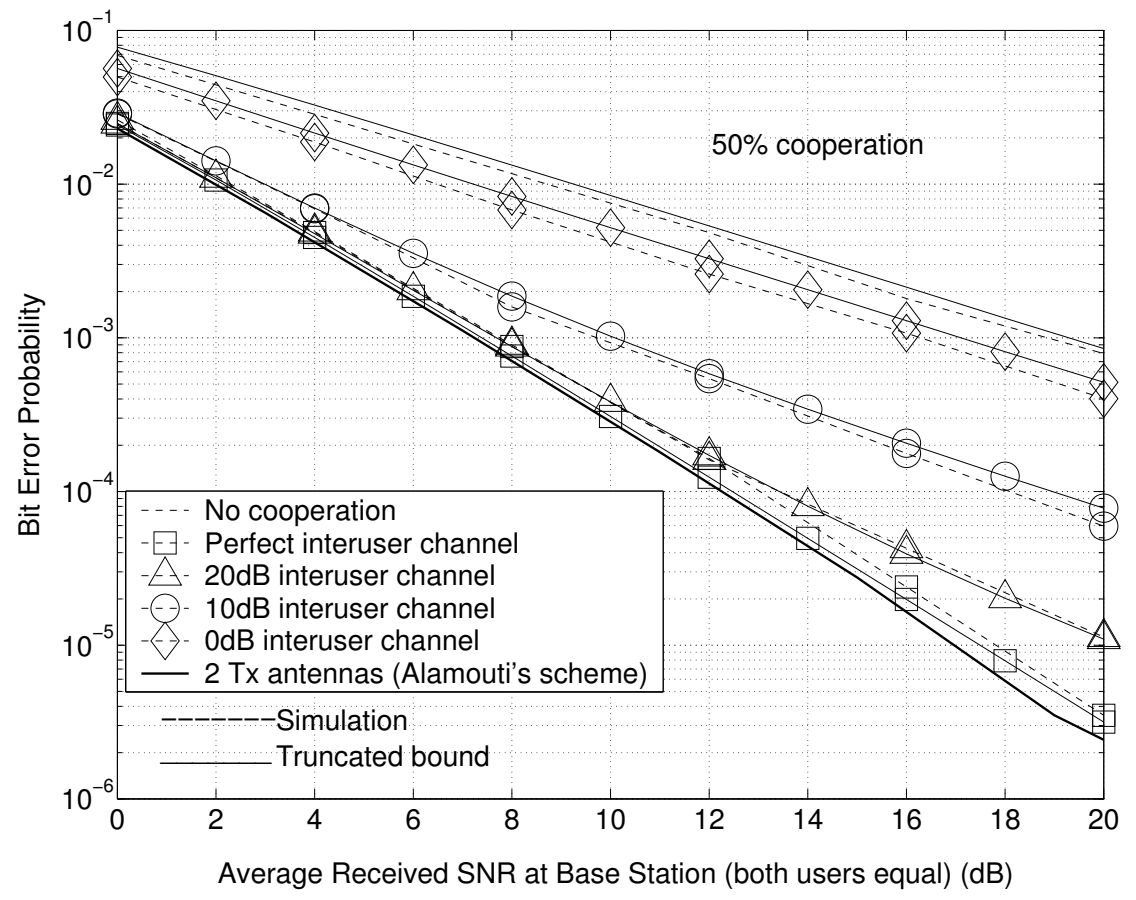

Fig. 5. Performance in slow Rayleigh fading with 50\% cooperation, equal uplink SNR, and reciprocal inter-user channels. 


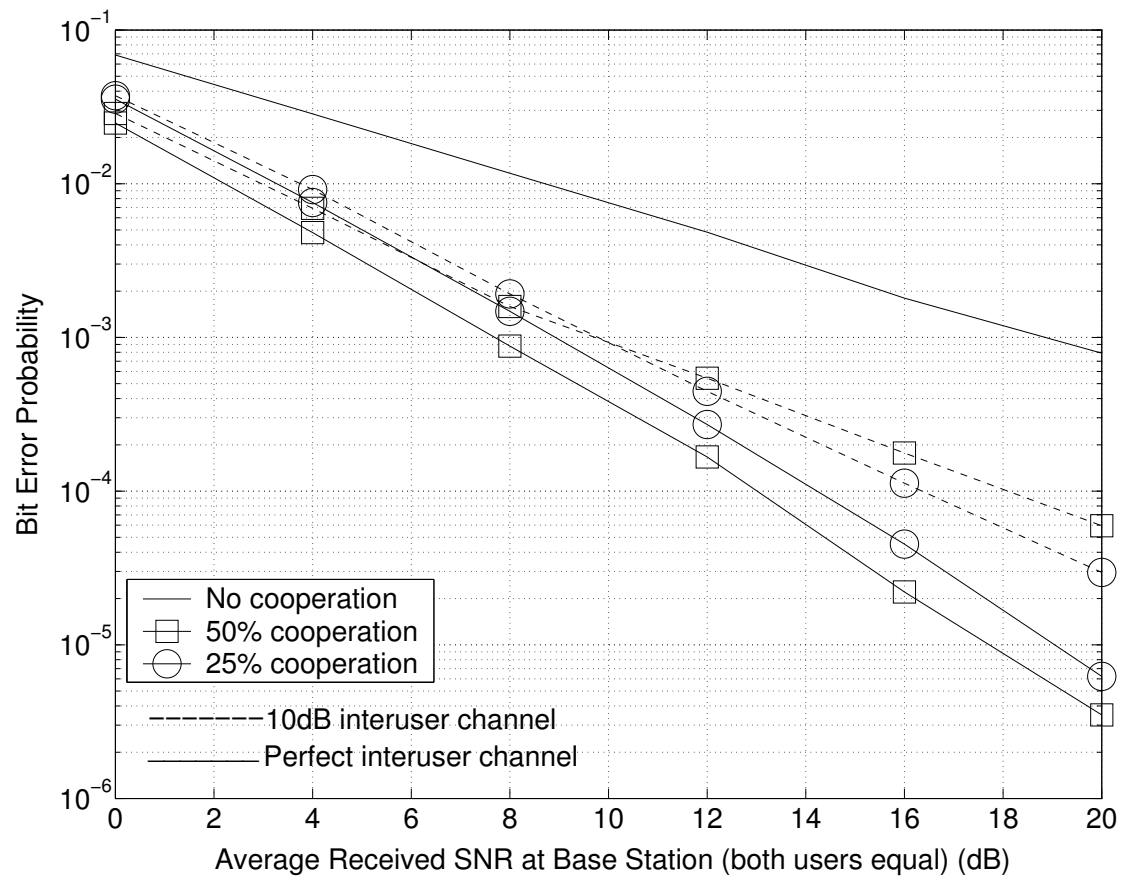

Fig. 6. Comparison of $50 \%$ and $25 \%$ cooperation in slow Rayleigh fading, equal uplink SNR. 


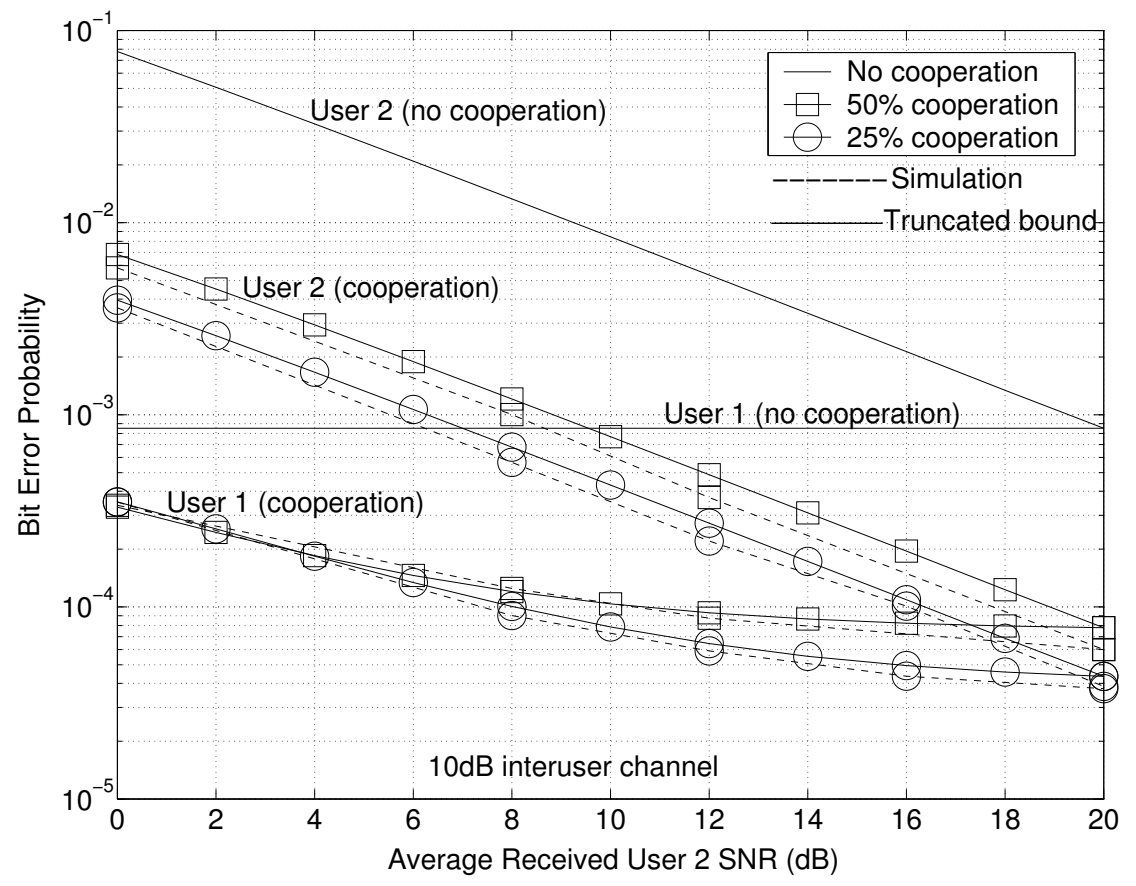

Fig. 7. Performance under asymmetric uplink conditions in slow Rayleigh fading. 


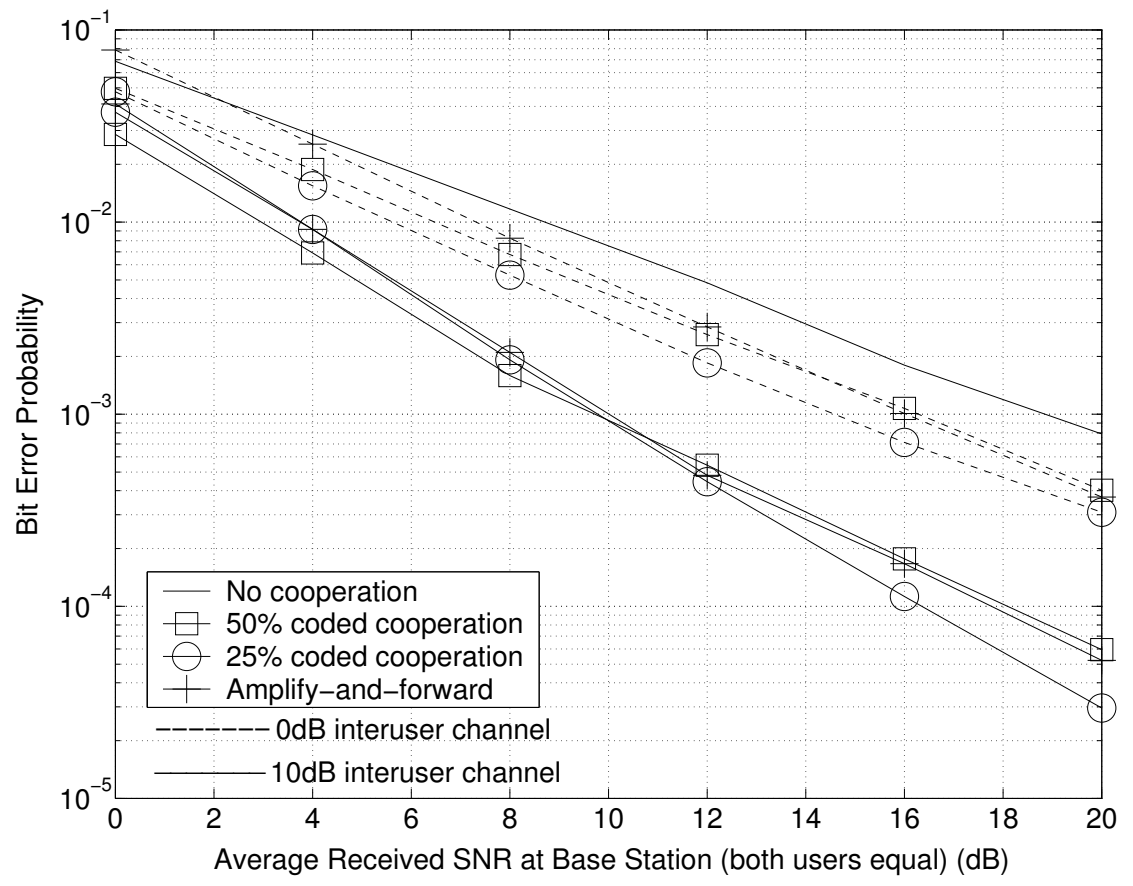

Fig. 8. Comparison of coded cooperation with amplify-and-forward under slow Rayleigh fading. 


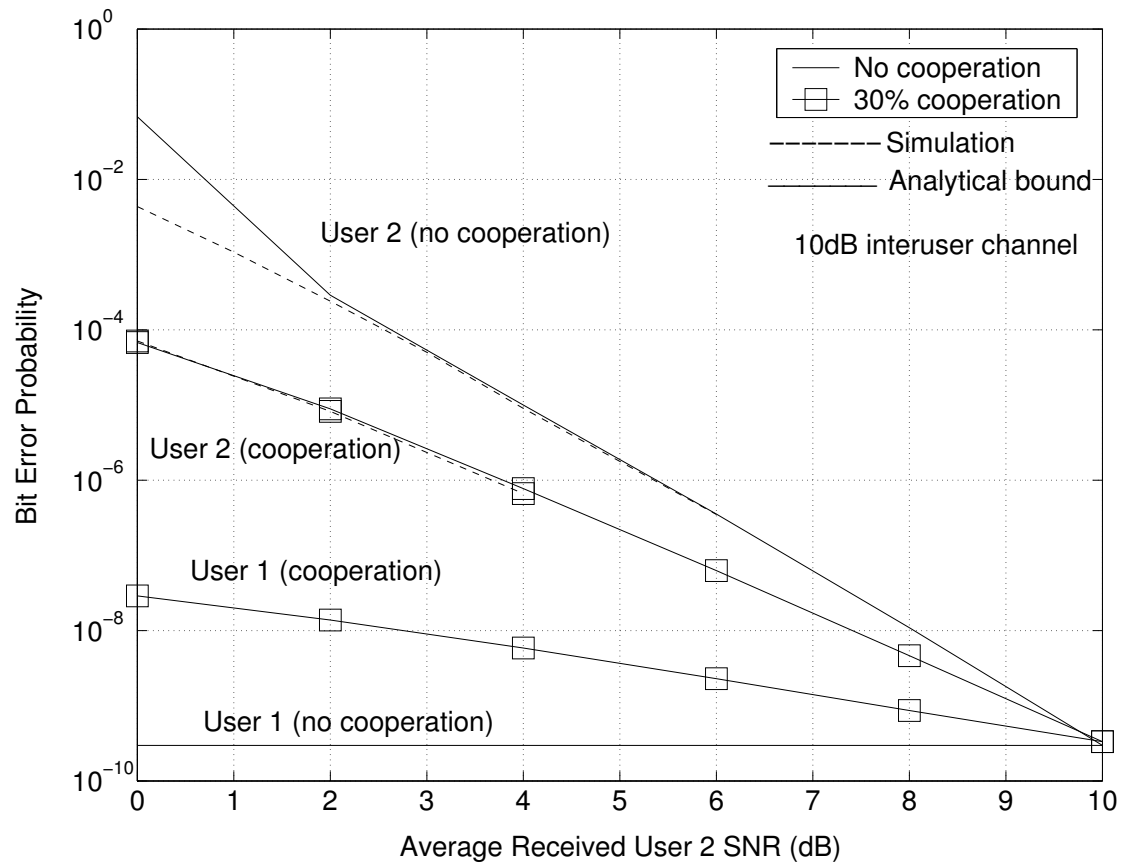

Fig. 9. Fast i.i.d. Rayleigh fading, unequal uplink average SNR, and independent inter-user channels (non-reciprocal). 

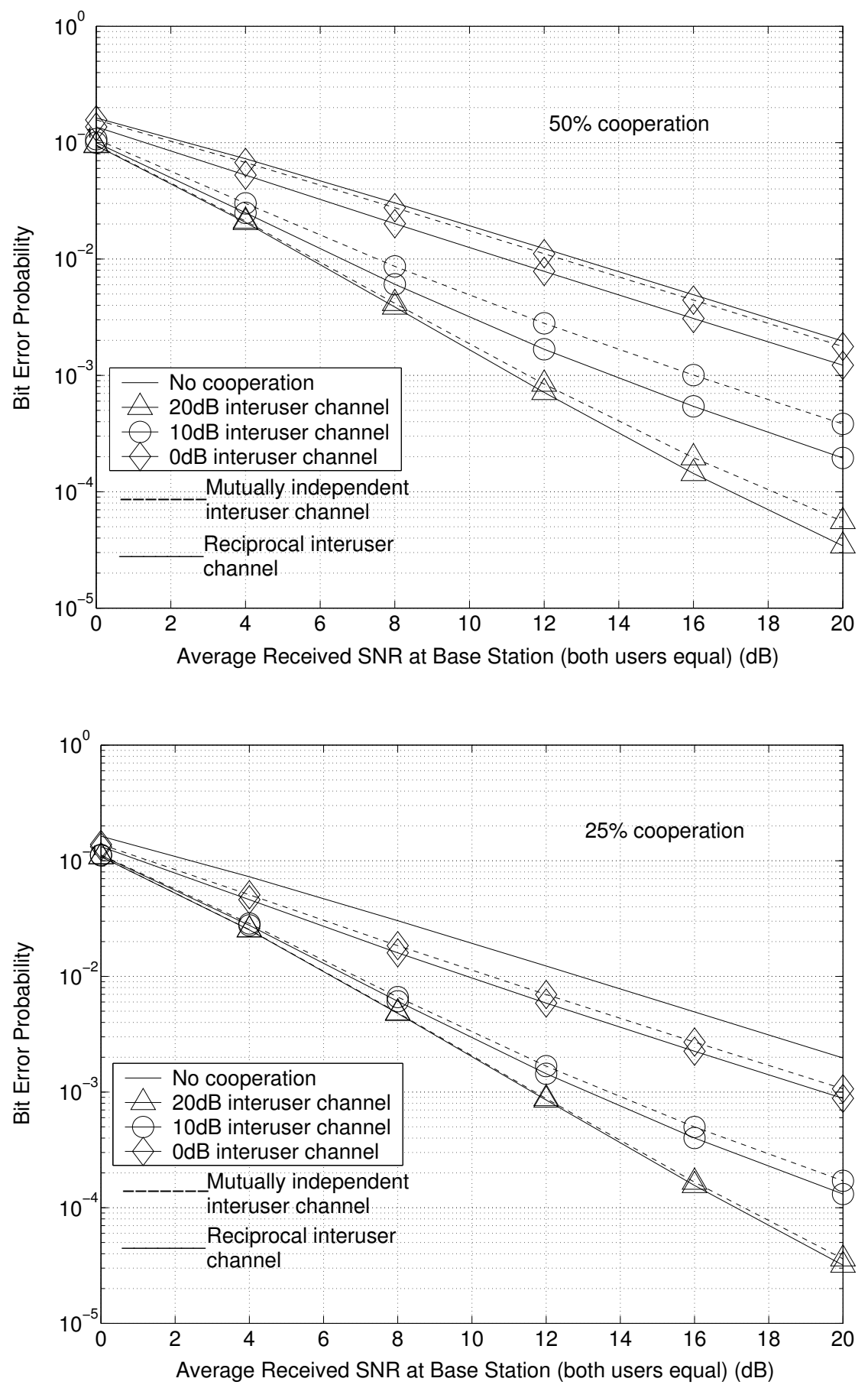

Fig. 10. Comparison (analytical bound) of coded cooperation for reciprocal and mutually independent inter-user channels of various qualities. 


\section{TABLE I}

COMPleXity FACTORS For END-TO-END BLER $10^{-2}, 50 \%$ COOPERATION, ANd RECIPROCAL INTER-USER CHANNEL

\begin{tabular}{|c|c|c|c|c|c|}
\hline \multirow{2}{*}{$\begin{array}{c}\text { Inter-user } \\
\text { Average SNR }\end{array}$} & \multicolumn{4}{|c|}{ Case Probability } & \multirow{2}{*}{$\begin{array}{c}\text { Complexity } \\
\text { Factor } k\end{array}$} \\
\hline & $\Theta=1$ & $\Theta=2$ & $\Theta=3$ & $\Theta=4$ & \\
\hline $20 \mathrm{~dB}$ & 0.992 & 0.0069 & 0.0007 & 0.0007 & 1.04 \\
\hline $10 \mathrm{~dB}$ & 0.92 & 0.067 & 0.0066 & 0.0066 & 1.13 \\
\hline $0 \mathrm{~dB}$ & 0.44 & 0.50 & 0.03 & 0.03 & 1.61 \\
\hline
\end{tabular}

